\title{
LA ESTRELLA, UN BOLICHE EN MEDIO DEL CAMINO (1884-1946, DEPTO. RANCUL, LA PAMPA)
}

\author{
Carlos Landa ${ }^{1}$, Virginia Pineau ${ }^{2}$, Luis $\mathrm{Coll}^{3}$, \\ Astrid Rearte ${ }^{4}$, Raúl Doro ${ }^{5}$ y Pilar Martínez ${ }^{6}$
}

Recibido: 5 de febrero de 2019. Aceptado: 31 de marzo de 2019

\begin{abstract}
Resumen
Entre 1885 y 1930, luego de las campañas militares contra los pueblos indígenas, el norte de la actual provincia de La Pampa comenzó a ser ocupado por instalaciones relacionadas con la explotación rural: estancias con sistema arrendatario, colonias, poblados y asentamientos comerciales. Desde 2008 nos encontramos estudiando esta problemática que se enmarca dentro de la denominada arqueología rural. En este trabajo se presentarán los avances preliminares con respecto al abordaje del sitio "Casa de Negocios La Estrella" (1884-1946) ubicado en las proximidades de la ciudad de Parera (Departamento de Rancul, provincia de La Pampa). Dicho sitio se trata de una pulpería o boliche que se hallaba en una intersección de caminos que lo vinculaba con diversos tipos de asentamientos (estancias, puestos y otros almacenes y casas de negocios) conformando parte constitutivo del paisaje rural reciente. En la campaña 2018 se realizó la determinación de la extensión del sitio, se recolectaron sistemáticamente los materiales de superficie y se realizaron 14 sondeos. Se comenzará con el análisis de mapas históricos a través del GIS, luego se explicitará la metodología de campo utilizada y finalmente se describirán los procesos de formación de sitio que afectaron a los materiales recuperados en los sondeos.
\end{abstract}

Palabras clave: Casa de Negocios La Estrella, Arqueología Rural, SIG, Materialidad

\section{Abstract}

Between 1885 and 1930, after the military campaigns against the indigenous peoples, the north of the current province of La Pampa began to be occupied by settlements related to rural exploitation: estancias, colonies, towns and commercial settlements. Since 2008 we are studying this problem that is part of the so-called rural archeology. In this paper we will present the preliminary advances in the study of the archeological site "La Estrella" (1884-1946) located in the vicinity of Parera (Department of Rancul, province of La Pampa). This site is a

1 Universidad de Buenos Aires, Facultad de Filosofia y Letras, Instituto de Arqueología/CONICET 25 de mayo $2213^{\circ} \mathrm{CABA}$. E-mail: carlosglanda@gmail.com

2 Universidad de Buenos Aires, Facultad de Filosofia y Letras, Instituto de Arqueología 25 de mayo $2213^{\circ}$ CABA. E-mail: virpineau@gmail.com

3 IDECU/CONICET Moreno 350 CABA. E-mail: sinuhe1979@yahoo.com.ar

4 Universidad de Buenos Aires, Facultad de Filosofia y Letras, Instituto de Arqueología 25 de mayo $2213^{\circ}$ CABA. E-mail: rearte125@gmail.com

5 Universidad de Buenos Aires, Facultad de Filosofia y Letras Puan 480 CABA. E-mail: ra.doro@ hotmail.com

6 Universidad Nacional de La Plata, Facultad de Ciencias Naturales y Museo, Laboratorio de análisis cerámico Calle 64 3, La Plata, Provincia de Buenos Aires. E-mail: alapilar@hotmail.com 
pulperia that was at an intersection of roads that linked it with various types of settlements (ranches, stalls and other stores) forming a constituent part of the recent rural landscape. In the 2018 archeological campaign the extension of the site's area was determined, the surface materials were systematically collected and 14 surveys diggings were made. This paper presents the analysis of historical maps through the GIS, the field methodology used and the site formation processes that affected the materials recovered in the surveys diggings.

Key Words: La Estrella warehouse, GIS, rural archaeology, materiality

\section{Introducción}

Durante los años comprendidos entre 1885 y 1930, a posteriori de las campañas militares contra los pueblos originarios pampeano-patagónicos, el norte de la actual provincia de La Pampa comenzó a ser ocupado por asentamientos vinculados a la explotación rural: estancias con sistema arrendatario, colonias, poblados y asentamientos comerciales. Desde el año 2008 nos encontramos estudiando este proceso desde la perspectiva conocida como Arqueología histórica (sensu Orser 2000) y con mayor especificidad dentro del marco de la denominada arqueología rural (Brittez 2002, 2004). Siguiendo a Brittez (2018) comprendemos a la Arqueología rural como un campo que queda definido por los objetivos que persigue y no necesariamente por el ámbito donde se la práctica. Dichos objetivos buscan abordar problemáticas tales como la expansión del capitalismo sobre las áreas rurales, el cambio social y sus formas, la constitución de nuevas clases sociales y el rol jugado por la materialidad, entre otras.

En este trabajo se presentan los avances preliminares con respecto al abordaje del sitio "La Estrella" (1884-1946) ubicado en las proximidades de la ciudad de Parera (departamento de Rancul, provincia de La Pampa). Dicho sitio se trata de una pulpería o boliche que se hallaba en una intersección de caminos que lo vinculaba con diversos tipos de asentamientos (estancias, puestos y otros almacenes y casas de negocios) conformando parte constitutivo del paisaje rural reciente. E1 presente trabajo se desarrolla en el marco del proyecto Ubacyt 01/Q496: "Abordaje arqueológico de las ocupaciones humanas en el norte y suroeste de La Pampa. Estrategias culturales en un paisaje de tierras áridas" dirigido por la Dra. Alicia Tapia. Su objetivo general hace foco en la caracterización del proceso de construcción social del paisaje en el sector norte de la actual provincia de La Pampa, desde los últimos asentamientos ranquelinos y las primeras avanzadas militares (1876-1885) hasta el ocaso del sistema rural basado en las colonias y el arrendamiento (ca. 1930). Para ello es menester poder establecer las diferencias/semejanzas entre las estrategias de ocupación y uso social del espacio que implementaron cada uno de los diversos grupos sociales en el área de estudio. De esta forma son objetivos específicos de este escrito:

Apreciar a escala local y diacrónica las continuidades y rupturas en torno al paisaje del sitio Casa de negocio La Estrella

Presentar los resultados de las primeras aproximaciones arqueológicas a dicho sitio

\section{Casa de negocios La Estrella 1884- 1946: primeras aproximaciones}

Durante el año 2013 hemos realizado la primera visita al sitio arqueológico Casa de Negocios La Estrella. Como mencionamos 


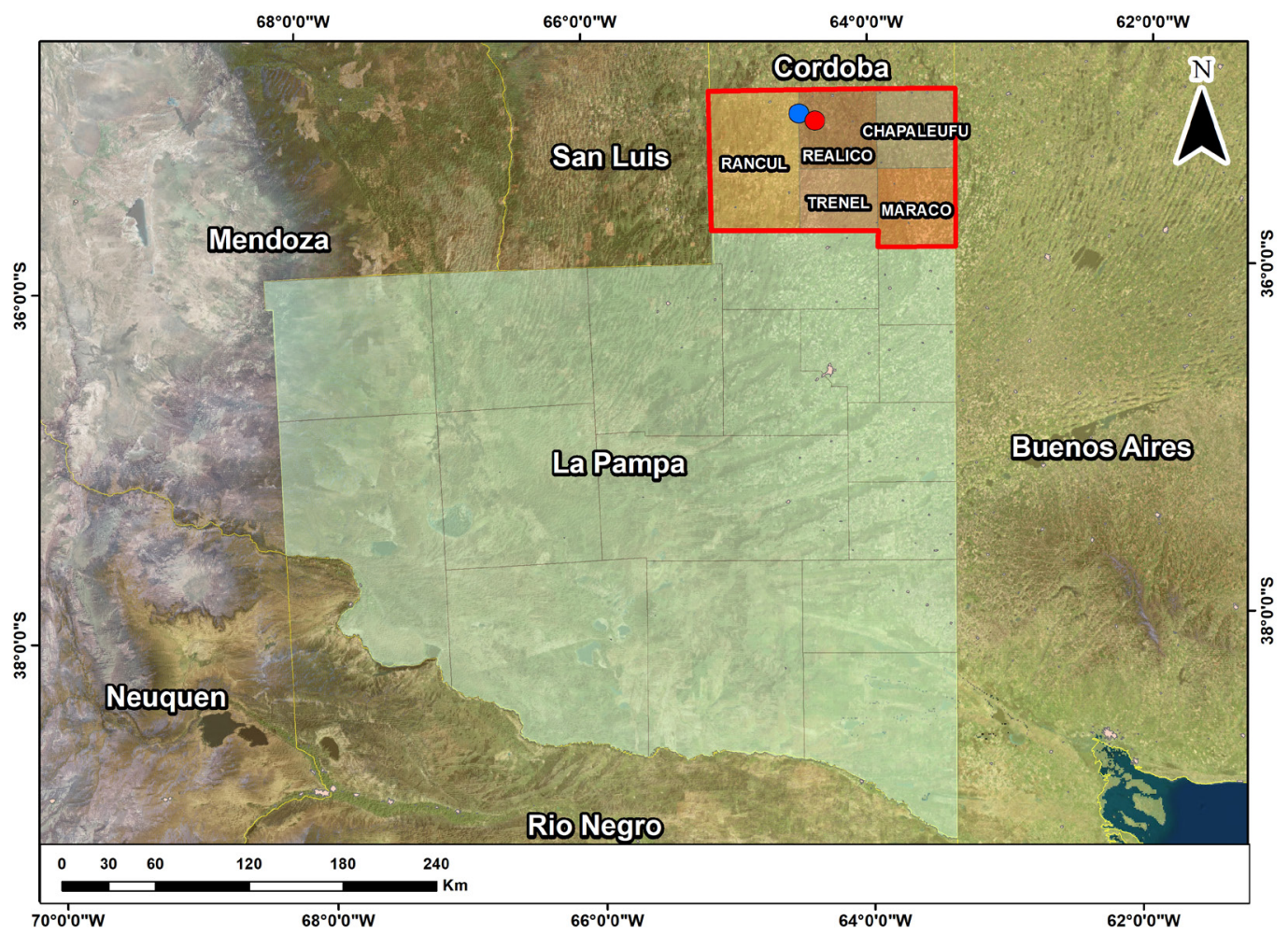

Figura 1. Mapa de región de estudio. El punto rojo representa la ubicación del sitio "Casa de negocios

La Estrella". El mismo se encuentra a $14 \mathrm{~km}$ al este del actual pueblo de Parera (en azul). Las imágenes satelitales Landsat 8 fueron obtenidas de USGS (https://earthexplorer.usgs.gov)

con anterioridad, el mismo se trata de una casa de negocios que funcionó desde fines del siglo XIX, cercana a la localidad de $\mathrm{Pa}-$ rera $\left(35^{\circ} 10^{\prime} 48,61^{\prime \prime} \mathrm{LS}-6^{\circ} 22\right.$ '26,04'LO), en el Departamento Rancul (Figura 1). Actualmente no se encuentran estructuras en superficie, aunque si existe cartelería identificatoria colocada por la municipalidad de dicha ciudad, que da cuenta sobre la historia del lugar. En aquella oportunidad, recorrimos el área y apreciamos en superficie numerosos materiales de vidrio, gres y metal. Procedimos también a georreferenciar el sitio. En el año 2014 efectuamos un primer sondeo exploratorio, a partir del cual se recuperó una botella de vidrio transparente completa. En un segundo reconocimiento en 2016 se llevó a cabo una prospección que posibilitó determinar la extensión del sitio a partir de los materiales en superficie. Lamentablemente, debido a las reiteradas inundaciones que imposibilitaron el acceso y los trabajos en el sitio no hemos podido continuar con las labores arqueológicas hasta enero de 2018, sus resultados se explicitan en acápites posteriores.

El almacén o boliche "La Estrella" es un sitio de particular interés para nuestra área de estudio, dado que se encuentra sobre el mismo camino en el cual se hallan el sitio Posta El Caldén o Casa de negocios Bor- 

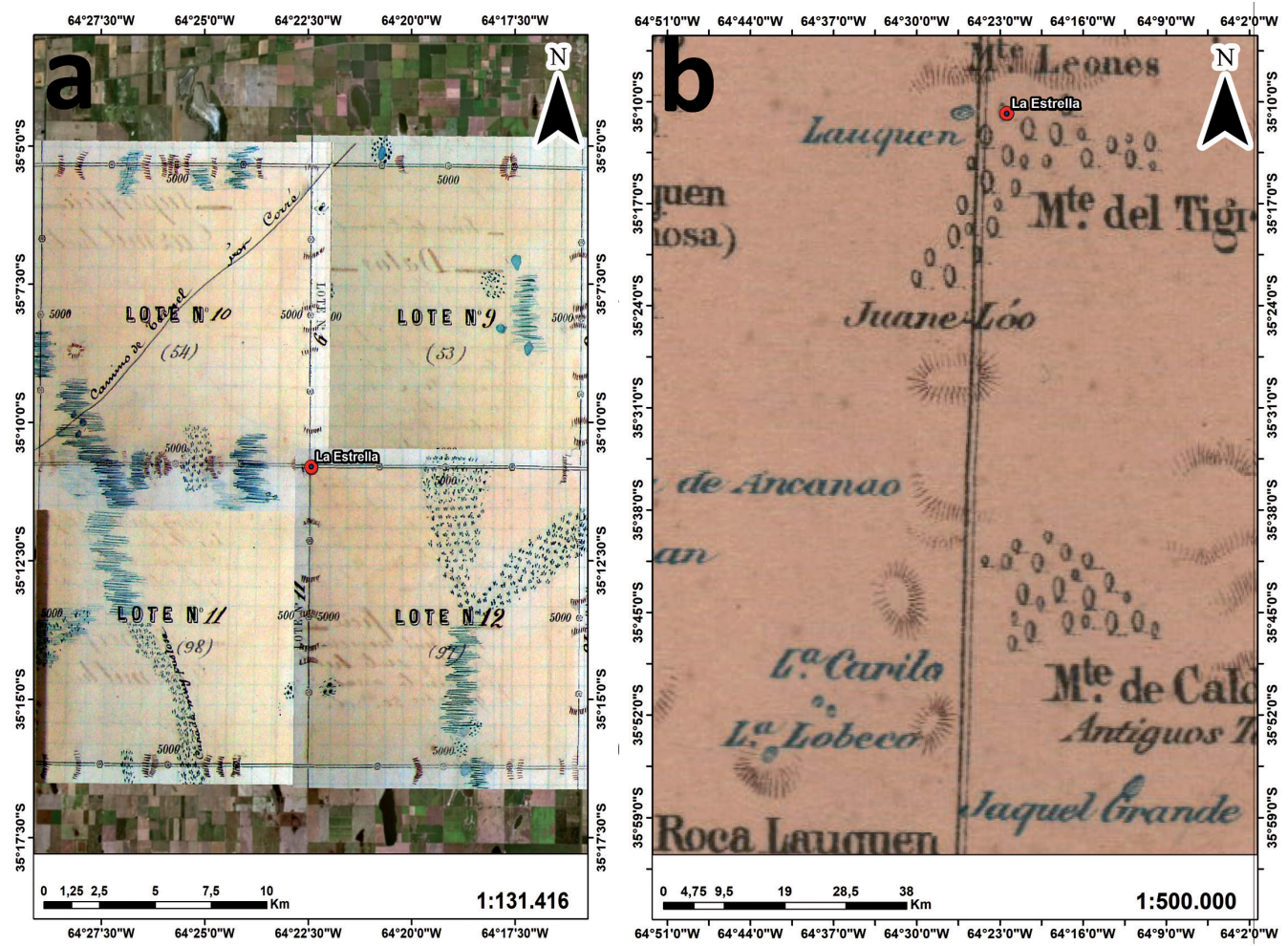

Figura 2. Ubicación de "La Estrella" en distintos corpus cartográficos. a: Dominguez 1882 (Libros Azules); b: Seelstrang 1886; c: Soldán 1888

darampé (Montanari et al. 2013; Pineau et al. 2014) y otra casa de negocios detectada en la cartografia histórica: "El Toro" (Coll y Landa 2018). Por otra parte, se trata de un asentamiento rural-comercial que coexistió con el corolario de la ocupación militar en la región (1879-1885). A su vez, también existen relatos escritos y orales que sostienen la coexistencia en el sitio de algunos miembros de comunidades originarias y criollos. Estas características convierten a "La Estrella" en un sitio liminal entre los espacios de frontera y la génesis del nuevo mundo rural. Dichos relatos de memoria oral fueron recopilados por el $\mathrm{Sr}$. Omar Agustín Rodríguez, Director del Museo del Tordillo de Parera. En ellos se da cuenta de la muerte de un niño (primer entierro del cementerio de Parera), previamente tratado por una machi ranquel en 1884. El sitio habria sido abandonado en 1946 y trasladado hacia la esquina diagonal opuesta del cruce de caminos subsistiendo hasta bien avanzada la década de 1870 .

\section{"La Estrella" y el espacio: representaciones cartográficas en la diacronia}

El quehacer cartográfico ha generado mapas que son el producto de maneras de imaginar, pensar y concebir el espacio social en forma sincrónica. Por ende, el re- 


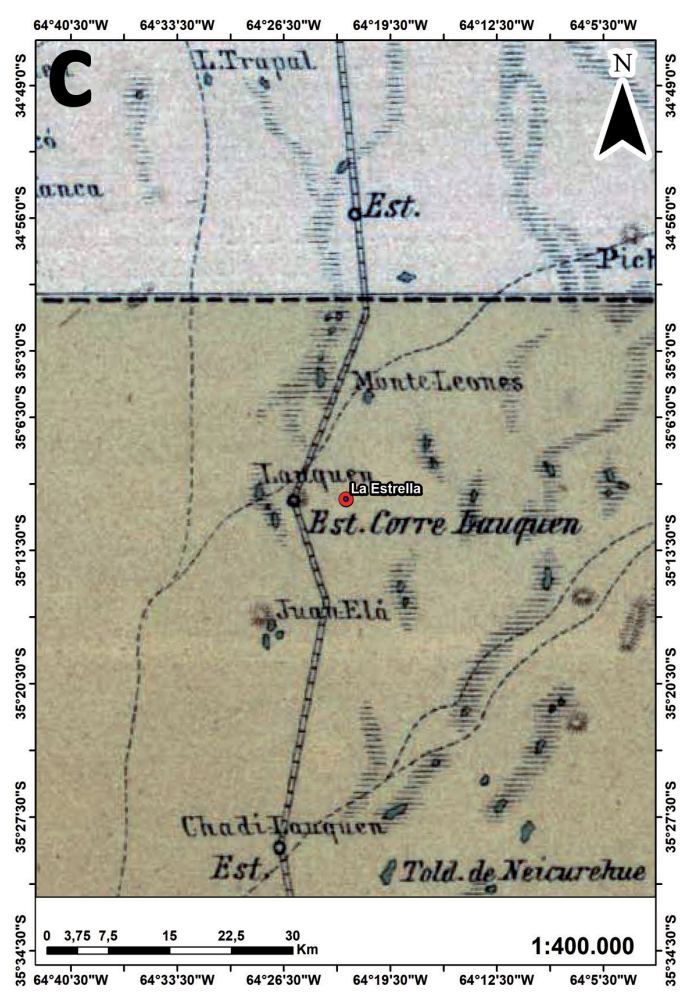

Figura 2. Continuación

levamiento de fuentes cartográficas de un periodo histórico posibilita llevar a cabo análisis en torno a las distintas formas de organizar, pensar y relatar el espacio de un determinado lugar a través del tiempo. Una sumatoria de complejas sincronicidades que permiten vislumbrar los cambios y continuidades espaciales en la diacronía.

Con el fin de poder desarrollar un análisis espacial que nos permitiera comprender diacrónicamente en las representaciones cartográficas del devenir del sitio casa de negocios "La Estrella" (sus vinculaciones con caminos, poblados y recursos hídricos) relevamos un acotado corpus documental hallado en la Dirección de Catastro de la Provincia de La Pampa (DGCLP, ciudad de Santa Rosa), en el Instituto Geográfico Nacional (IGN) y en bibliotecas personales. Dicho corpus cartográfico se encuentra constituido por mensuras, atlas nacionales y mapas provinciales, fueron desarrolladas tanto por los primeros agrimensores de la región a pedido del Estado o de particulares, abarcando un periodo temporal comprendido entre los años 1882 y 1930. Se hace hincapié en los aspectos cualitativos de las mismas y sus cambios en la diacronía (vías de comunicación, características geomorfológicas y ambientales, entre otros). Para efectuar dicho análisis se utilizan Sistemas de Información Geográficos (SIG), específicamente el software libre QSIG. Las fuentes cartográficas históricas relevadas fueron:

\section{-Libros Azules (1882)}

Este corpus cartográfico constituye la primera agrimensura del territorio a pedido de la Nación. La misma estuvo a cargo de Joaquín Domínguez (DGCLP). En ella no aparece representada la Casa de Negocios "La Estrella" (no hemos hallado al momento datos históricos anteriores al año 1884. La georreferenciación tomada in situ posibilitó ubicarlo en dicha mensura en la intersección entre los lotes 10, 9, 12 y 11 del sector 1 A del loteo provincial. Se encontraría a $9 \mathrm{~km}$ al $\mathrm{SE}$, a distancia ideal más corta, del camino de Trenel a Luan Carré (rastrillada indígena que atraviesa la región en orientación SO-NE). En el punto en donde se ubicaría el futuro sitio se encuentra representado un médano (existe una cadena de médanos próxima hacia el $\mathrm{N})$ y a $2 \mathrm{~km}$ al E de un gran bañado (Figura 2a).

\section{-Arturo Seelstrang (1886)}

Este mapa fue desarrollado por el agrimensor Arturo Seeltrang en el año 1886 como parte de un atlas de la República. Pese a contar con documentación histórica en torno a su existencia, al igual que en el 
caso anterior, en este mapa no se encuentra representada la Casa de negocios "La Estrella". Su ubicación estaría a unos 6 $\mathrm{km}$ al E de la estación proyectada de ferrocarril Corre Lauquen (posiblemente dicha proyección se haya llevado a cabo sobre un anterior camino militar de orientación $\mathrm{N}-\mathrm{S})$. Mantiene la misma distancia con respecto al camino mencionado en los Libros Azules como de Trenel a CarreLauquén. Se encuentra a $9 \mathrm{~km}$ al $\mathrm{E}$ de la laguna Lauquen (topónimo que significa laguna en Mapudungun) y a $25 \mathrm{~km}$ aproximadamente del topónimo Juan Elá (representado por un médano y tres lagunas). Asimismo, se encuentra a una distancia aproximada de $70 \mathrm{~km}$ de los toldos de Neicorehué (topónimo que significa "lugar de reunión" en lengua Mapudugun) (Figura 2b).

-Mariano Felipe Paz Soldán (1888)

$\mathrm{Su}$ autor fue Mariano Felipe Paz Soldán. Confeccionado en 1888. Se trata de un libro que contiene los mapas de cada provincia y de los territorios nacionales, Atlas Geográfico de la República Argentina. Al igual que en los casos anteriores, en este mapa no se encuentra representada la Casa de negocios "La Estrella". La ubicación de dicho sitio se encontraría posicionada al sur del Monte del Tigre y próximo al monte Leones (aproximadamente $10 \mathrm{~km}$ al N). El punto en donde se encontraría "La Estrella" se localiza a $3,5 \mathrm{~km}$ al O aproximadamente de una línea férrea proyectada (consignado en 1886 en el atlas de Seelstrang) y a unos $6,5 \mathrm{~km}$ al $\mathrm{O}$ aproximadamente del topónimo Lauquen (laguna en Mapudungun, la misma está representada). Se hallaría también a $38 \mathrm{~km}$ aproximadamente al $\mathrm{S}$ de un médano cuyo topónimo denominado Juane-Lóo (médano de Juane) y a $65 \mathrm{~km}$ aproximadamente al $\mathrm{S}$ del monte de Caldera (sitio en donde se menciona la existencia de antiguos toldos y el Jagüel Grande) (Figura 2c).

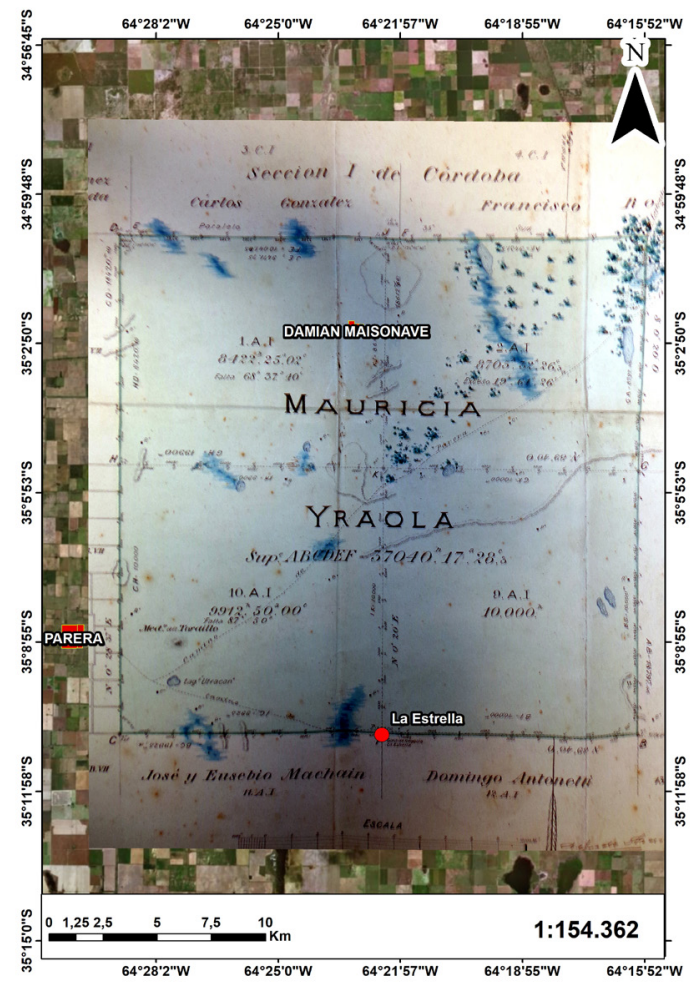

Figura 3. Ubicación de "La Estrella" en Thamm 1899 (Carpetas Amarillas)

\section{-Carpetas Amarillas (1899)}

Esta documentación constituye una serie de agrimensuras realizadas con el fin de subdividir lotes a pedido de sus nuevos dueños. Las mismas estuvieron a cargo del ingeniero Alfredo Thamm y se realizaron entre los años 1886 a 1902. En una de ellas (propiedad Iraola, año 1899, Sección 1 Fracción A Lotes 1, 2, 9 y 10, DGCLP) el sitio aparece representado bajo la denominación de Casa de negocio "La Estrella", ubicándose en el camino a Parera (E-O). Por otra parte, se encuentra a $1,3 \mathrm{~km}$ al E del bañado registrado en Libros Azules o del topónimo Lauquen representado en los mapas anteriores. Asimismo, se halla próximo a varios puestos rurales (el más 
cercano unos $700 \mathrm{~m}$ al NE). Cabe destacar que a $10 \mathrm{~km}$ al NO se ubica la laguna Utracan (empinado o invernada) y en mismo sentido a 10,5 km del médano El Tordillo. Por último, "La Estrella" se encuentra a 14 km del pueblo de Parera (Figura 3).

\section{-Chapeaurouge (1901)}

Este documento se trata de un Atlas del Plano Catastral de la República Argentina confeccionado por el agrimensor Carlos de Chapeaurouge en 1901. Los mapas que lo componen cuentan con la división catastral de la época. Si bien las Carpetas amarillas (1899) ya presentan la existencia de la Casa de negocio "La Estrella" esta cartografía no da cuenta de su existencia. Esto puede deberse a que la base para realizar dicho Atlas fueron los Libros Azules (1882). Es comprensible pensar que se haya utilizado la cartografia de los agrimensores nacionales Domínguez y Maqueda dado que las carpetas amarillas no abarcan la totalidad de la provincia de La Pampa pues dichos trabajos catastrales se realizaron a pedido de los propietarios de las tierras. La ubicación georreferenciada del sitio se halla a una distancia aproximada de $10 \mathrm{~km}$ SO del Monte de los Leones (topónimo que se repite en mapas anteriores) y a $7 \mathrm{~km}$ al $\mathrm{E}$ de Lauquen y $9 \mathrm{~km}$ al $\mathrm{S}$ del camino de Trenel por Carre Lauquen (rastrillada indígena) (Figura 4a). Además, se observa una línea férrea proyectada a aproximadamente a $14 \mathrm{~km}$ al E de la localización de la Casa de Negocios La Estrella.

\section{-Manuel Farinos (1919)}

Se trata del Mapa del Territorio de La Pampa confeccionado por el agrimensor Manuel Farinos en 1919 a una escala de 1:400000. Es un mapa político administrativo. Cuenta con datos catastrales, demográficos, ambientales (clima, flora y fauna), agropecuarios, comerciales, caminos y vías férreas. El sitio Casa de Negocios La Estrella no aparece representado. La ubi- cación de su georreferenciación estaría a 2,4 $\mathrm{km}$ al $\mathrm{E}$ de un camino rural que conecta los pueblos de Parera con Falucho. Mantiene la misma distancia estipulada en el mapa anterior de $14 \mathrm{~km}$ con respecto a Parera (Figura 4b).

\section{-Lefrancois y Porri (1930)}

Mapa del Territorio Nacional de La Pampa: el mismo fue confeccionado por los ingenieros Alberto Lefrancois y Pablo Porri en 1930 y dibujado a una escala de 1:400000. Cuenta con divisiones catastrales, estancias, localidades, colonias, caminos, vías férreas, médanos y sierras. Al igual que en el mapa anterior tampoco se encuentra representado el sitio Casa de Negocios La Estrella. La ubicación de la georreferencia estaría próxima a un camino rural $(2 \mathrm{~km}$ al E) que conecta la colonia Yanquetrú con la colonia La María. Se encuentra aledaño a la colonia La Alfalfa y a unos $18 \mathrm{~km}$ al NE del pueblo La Alfalfa (pueblo actualmente inexistente) (Figura 4c).

Descripto el corpus documental analizaremos a escala local y diacrónica las continuidades y rupturas en torno al paisaje del sitio Casa de negocio "La Estrella". A pesar de que estas fuentes cartográficas contienen distintas escalas, resoluciones y temáticas (catastro, mapas provinciales y atlas); es posible apreciar cambios y persistencias en las distintas representaciones espaciales.

Un aspecto a destacar es que la Casa de negocios "La Estrella" solamente se encuentra representada en las Carpetas Amarillas. Esto es producto de que los mapas posteriores (provinciales y atlas) al poseer una mayor escala pierden resolución espacial, destacándose solo aquellos topónimos de mayor relevancia (poblados, estaciones de ferrocarril, colonias, etc.). Sin embargo, se conoce a través de la memoria oral colectiva que dicho espacio se encontró ocupado por este negocio hasta la década del 70 del siglo XX. Allende estas di- 
Figura 4. Ubicación de "La Estrella" en distintos corpus cartográficos. a: Chapeaurouge 1901; b: Farinos 1919; c: Lefrancois y Porri 1930
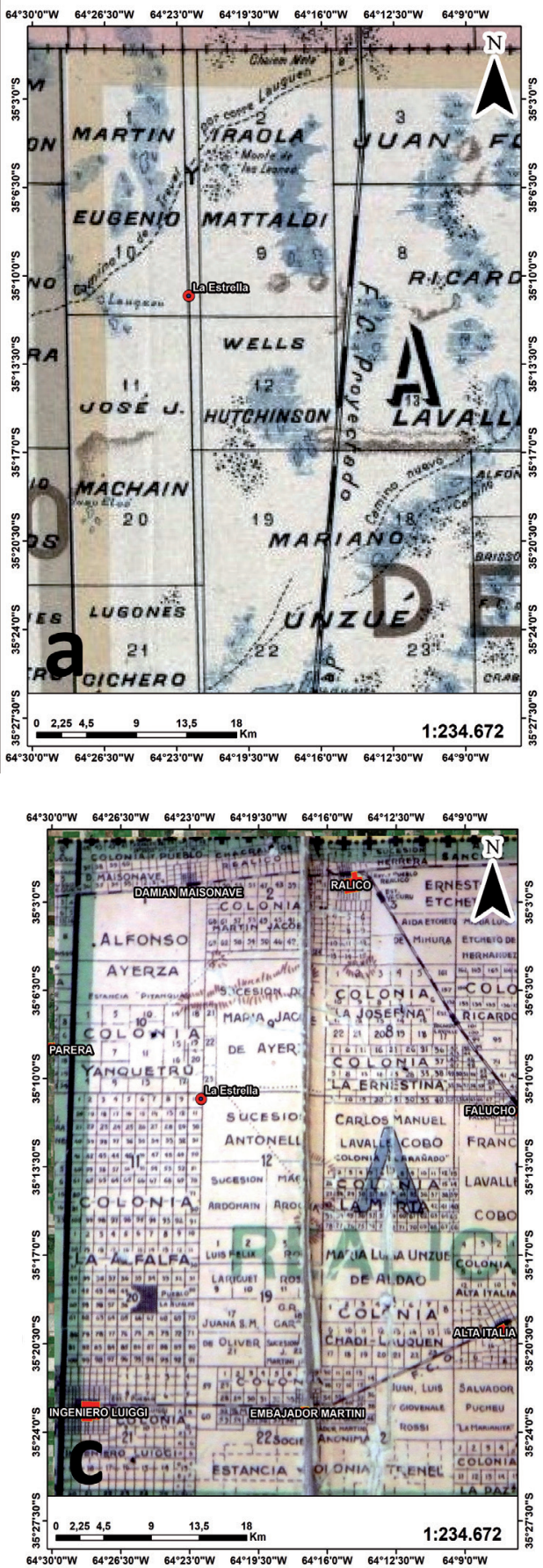

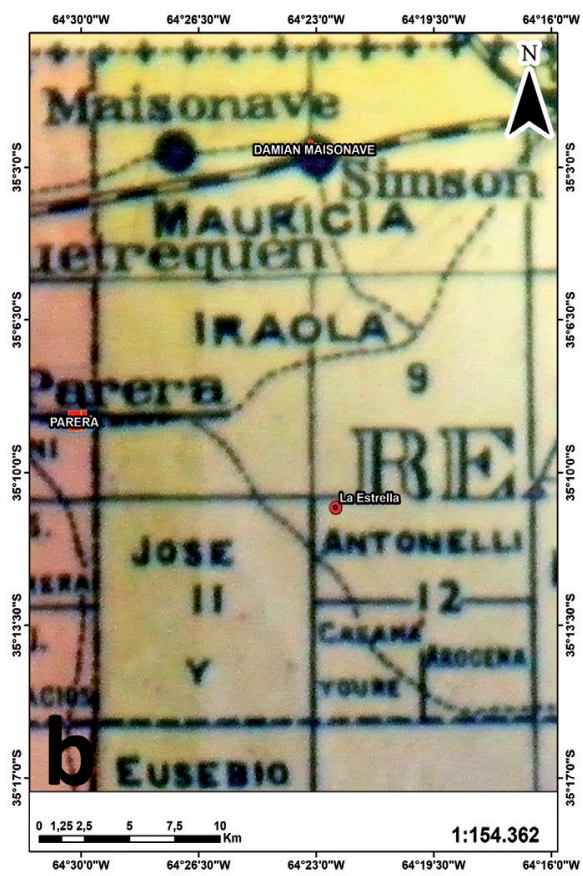


ferencias de escala y resolución, se puede estar observando ciertas continuidades en los rasgos tanto naturales como antrópicos (especialmente los caminos) del paisaje en las cercanías de la ubicación del sitio.

Por otra parte observamos que los recursos hídricos y montes de vegetación son representados en casi todos los mapas, con excepción de Farinos (1919) y Lefrancois y Porri (1930). Esta descripción de los recursos de la zona es crucial para comprender porque se encuentra proyectada una vía de ferrocarril en las cercanías de la casa de negocios. Esta línea es representada solamente en los mapas de Seelstrang (1886), Soldán (1888) y Chapeaurouge (1901). En este último la proyección del ferrocarril se encuentra al Este de la Estrella, a diferencia de los otros dos que la línea férrea iba a pasar cerca de la laguna de Lauquen.

En cuanto a los caminos se observa en las Carpetas Amarillas que la casa de negocios se encuentra localizada a la vera del camino de Parera a Villegas, conectando dicho lugar (Rappaport 2003) con estancias, puestos y poblados como La Marianita, Antigua Estancia Claudio Kirchner, Casa de Negocios Bordarampé y Cia. o El Toro, entre otros (Coll y Landa 2018). Sin embargo, no podemos distinguir en las representaciones de los mapas restantes la continuidad de este camino. Asimismo, se observa en el resto de la cartografia la continuidad en partes de la rastrillada indígena de Trenel a Luan Carré descripto en los Libros Azules; que luego será el camino que comunique los pueblos de Parera y Realicó pasando por las colonias de Yaquetru y Martín Jacob. De esta forma, se destaca que allende los cambios acaecidos en la diacronía, ciertos caminos continúan siendo utilizados a través de estos años (e.g. segmentos del camino de Parera a Villegas) (Coll y Landa 2018).

En sintesis, consideramos que la ubicación de la casa de negocios "La Estrella" se encuentra en un espacio estratégico, tanto por la cercanía de recursos naturales críticos (agua y madera) como de poblados, estancias, puesto y colonias, todas ellas interconectadas por vías de comunicación de diferente magnitud (antiguas rastrilladas y nuevos caminos rurales). Asimismo, estos distintos documentos cartográficos nos permitieron apreciar y comprender a escala de sitio -con las precauciones pertinentes-tanto las continuidades como los cambios en ciertos rasgos del paisaje.

En un futuro trabajo se llevarán a cabo comparaciones entre las diversas características del paisaje representadas en los distintos documentos cartográficos con las imágenes satelitales actuales (Google Earth) de la región y sitio en donde realizará las actividades arqueológicas. Dicha comparación posibilitará tanto la observación de las persistencias de los distintos rasgos espaciales en la actualidad como la planificación de nuevas investigaciones arqueológicas en asentamientos o rasgos vinculados.

\section{Trabajos de campo arqueológicos}

En la campaña de marzo de 2018 se realizó la determinación de la extensión del sitio. Se estableció un rectángulo de $40 \mathrm{~m}$ x 22 m teniendo en cuenta la dispersión de los materiales en superficie. Se trazaron 8 transectas de $22 \mathrm{~m}$ x $5 \mathrm{~m}$. Se recolectaron sistemáticamente con dos operadores que recorrieron las transectas en sentido este-oeste todos los materiales de superficie. Los materiales en superficie aumentan de norte a sur con dos áreas de concentraciones entre las transectas 2 a 4 y 6 a 8 con un total de 318 fragmentos recuperados. El 83\% (265:318) del total del material recuperado se corresponde con fragmentos de vidrio y el 8\% (24:318) con materiales de loza. El 9 \% (29:318) restante se distribuye entre materiales de construcción, metales y gres (Figura 5). 


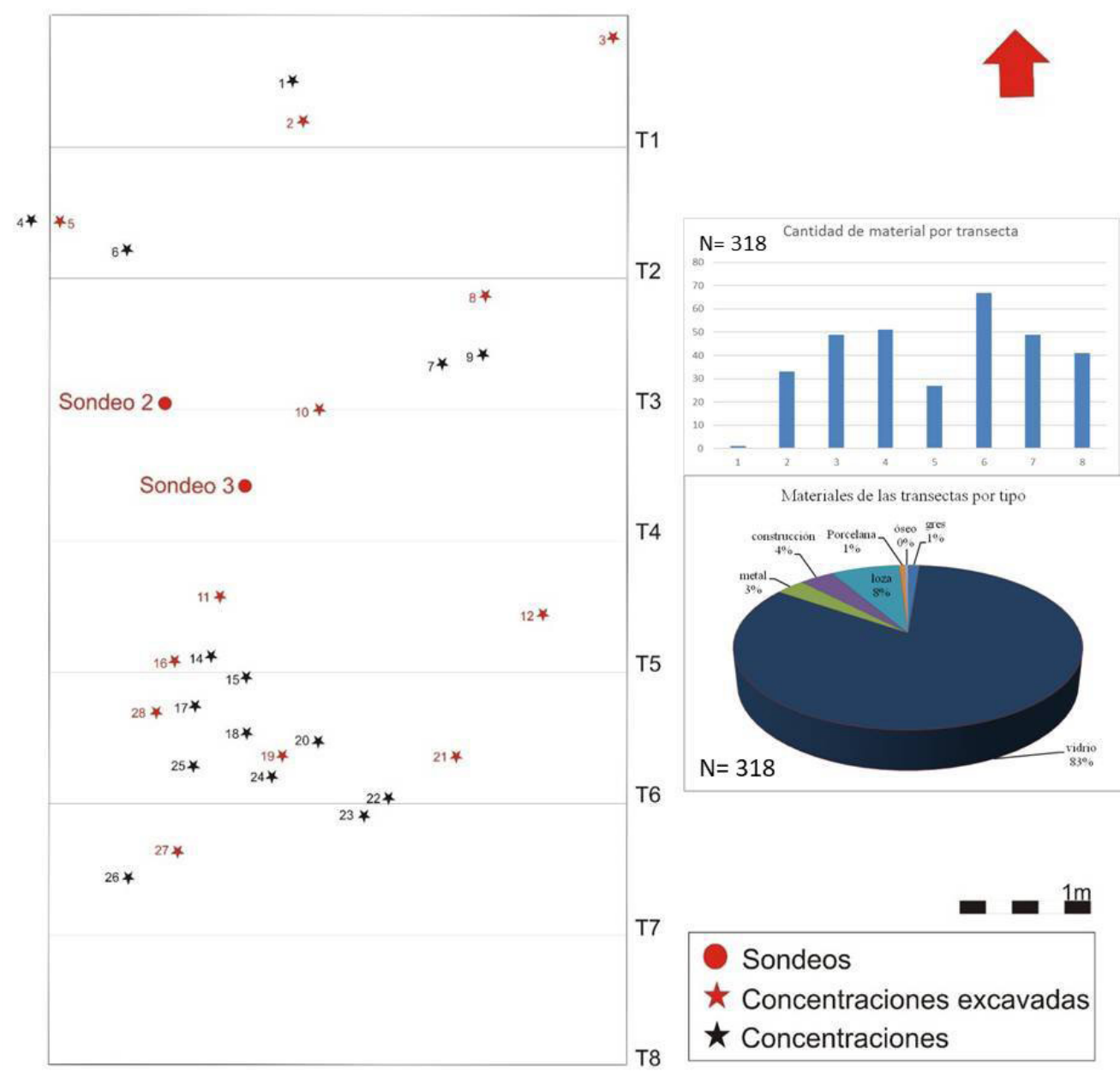

Figura 5. Área establecida de extensión del sitio Casa de Negocios La Estrella con las transectas determinadas. Los gráficos muestran la cantidad y tipo de los materiales recuperados en ellas.

Luego se realizó una prospección con un detector de metales sobre las transectas y se determinaron 28 áreas de concentración de materiales subsuperficiales. A partir de ellas se excavaron 12 sondeos de $1 \mathrm{~m} \times 1 \mathrm{~m}$ teniendo en cuenta las áreas de concentración de materiales superficiales y subsuperficiales. En 11 de los sondeos se recuperaron materiales similares a los provenientes de superficie. Todos los materiales $(\mathrm{N}=318)$ se recuperaron hasta los $35 \mathrm{~cm}$ de profundidad por lo que se corresponden 

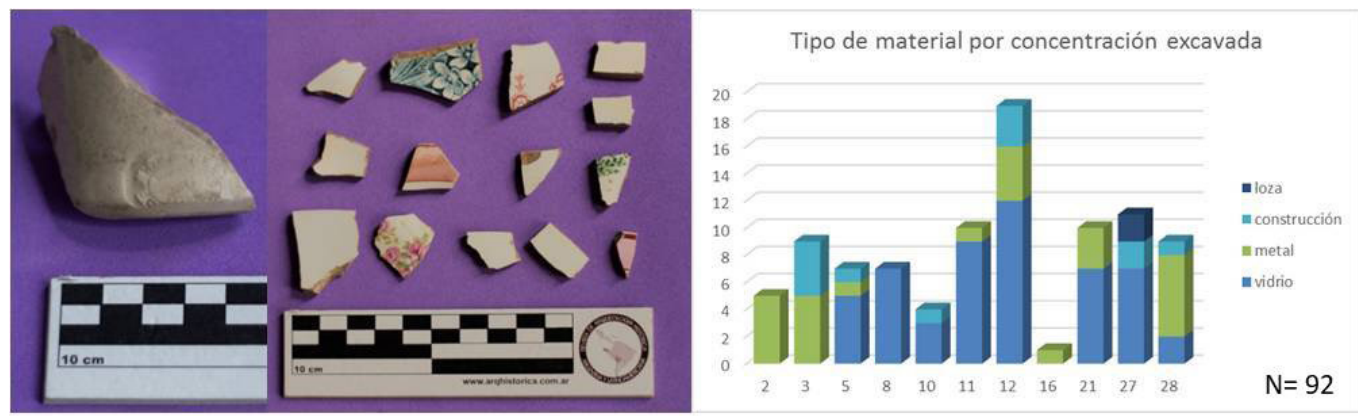

Figura 6. Tipos de materiales recuperados en las concentraciones excavadas. A la izquierda fragmento de botella de gres con sello H. Kennedy que fueron fabricadas entre 1866 y 1929 en Glasgow y que fueron utilizadas en nuestro país para contener diversas marcas de cervezas locales (Pineau y Andrade 2018)

con la alteración que provoca el arado de la tierra: fragmentación del material de superficie y enterramiento hasta los 30 $\mathrm{cm}$ aproximadamente. Hasta el momento no se han determinado áreas de descarte. Los materiales recuperados se corresponden con un asentamiento de fines del siglo XIX y principios del siglo XX (Figura 6).

Finalmente, se realizaron dos sondeos a fin de ubicar el sondeo realizado en 2014 en el que se recuperó una botella transparente completa. Estos sondeos tienen las mismas características que las descriptas previamente para los 12 sondeos anteriores. Dada la escasa información provista por las fuentes escrita y orales relevadas, consideramos que la Arqueología se torna una herramienta heurística primordial a la hora de generar conocimientos en torno a las prácticas sociales de los habitantes y al rol de la cultura material en un temprano sitio rural-comercial cuya ocupación fue de aproximadamente sesenta años de duración, tarea que emprenderemos en futuras campañas.

\section{A modo de cierre}

En este trabajo nos propusimos, en primer lugar, apreciar a escala local y diacrónica las continuidades y rupturas en torno al paisaje del sitio Casa de negocio La Estrella. Al respecto, se relevaron mapas históricos y mensuras desde 1882 (previo a la existencia de la Casa de negocio) hasta 1930. Solamente en la mensura de 1899 aparece la Casa de Negocios La Estrella. Esto es debido a la escala y al objetivo de la mensura, poder valuar esa fracción del terreno, posiblemente para su posterior venta. Con el Sistema de Información Geográfica se analizaron dichos mapas históricos y se pudieron observar cambios y continuidades en el ambiente cercano a La Estrella. En particular se pudo determinar que la Casa de Negocios estuvo ubicada en el cruce de caminos y bien abastecida de agua dulce y leña.

Posteriormente, nos propusimos presentar los resultados de las primeras aproximaciones arqueológicas a dicho sitio. Se 
realizaron las primeras tareas de determinación de la extensión del mismo así como también la recolección superficial de los materiales y la designación de áreas de excavación. Se excavaron 12 sondeos en los que se recuperó material de diversas materias primas adscribibles a finales del siglo XIX y principios del siglo XX. A futuro continuaremos ampliando las áreas de excavación a fin de recuperar las áreas de descarte de la Casa de Negocios.

\section{Bibliografia}

BRITTEZ, F. 2002. Investigaciones en arqueología rural: Sitio Vizcacheras (Partido de Coronel Brandsen, Provincia de Buenos Aires). Campañas 1998-1999. Arqueología Histórica Argentina. Actas del Primer Congreso Nacional de Arqueología Histórica, pp. 395-403. Editorial Corregidor, Buenos Aires.

BRITTEZ, F. 2004. Arqueología Rural en el partido de Coronel Brandsen, provincia de Buenos Aires. En La Región Pampeana -su pasado arqueológico. Actas del I Congreso de Arqueología de la Región Pampeana Argentina, editado por C. Gradin y F. Oliva (eds.), pp. 211-222. Laborde Editor, Rosario.

BRITTEZ, F. 2018. Introducción. Paisajes de la campaña pampeana (siglos XIX y XX). En Abordajes desde la Arqueologia rural en Argentina, editado por C. Landa, V. Pineau, E. Montanari y J. Doval (eds), pp. 5-20. South American Archaeology Series, Archaeopress, Oxford, Gran Bretaña.

COLL, L.J. y C.G. LANDA. 2018. La rayuela del camino (con un pie en la continuidad y otro en la ruptura). Un acercamiento inicial al análisis espacial de la dinámica de los caminos y asentamientos. Revista de Arqueología Histórica Ar- gentina y Latinoamericana 12:174-200. MONTANARI E.; C. LANDA y V. PINEAU. 2013. Caldén: pulpería y posta de caminos en el norte de La Pampa (fines del siglo XIX). Una primera aproximación. Revista Científica del Museo de La Plata. Volumen especial de Arqueología de la Región Pampeana:395-404.

PINEAU V.; E. MONTANARI; M. LUCCHETTA; F. CARETTI y C. LANDA. 2014. Prácticas de consumo de bebidas etílicas en una "casa de negocios" pampeana de fines del siglo XIX. Análisis de precintos de plomo y de fragmentos vitreos (Posta El Caldén, Departamento de Realicó, La Pampa). Revista del Museo de Antropología 7(1):55-64.

PINEAU, V. y A. ANDRADE. 2018. Los fragmentos de gres del sitio Mariano Miró. Análisis de su contenido y procedencia (La Pampa, siglo XX) Revista de Antropología del Museo de Entre Rios 4(2):111-121.

ORSER, C. 2000. Introducción a la Arqueología Histórica. Asociación Amigos del Instituto Nacional de Antropología y Pensamiento Latinoamericano (AINA), Buenos Aires.

RAPOPORT, A. 2003. Cultura, Arquitectura y Diseño. Ediciones Universitat Politécnica de Cataluña, Barcelona, España.

\section{Fuentes documentales:}

CHAPEAurouge, C. 1901. Atlas del Plano Catastral de la República Argentina. Eigendorf y Lesser.

DIRECCIÓN GENERAL DE CATASTRO DE LA PROVINCIA DE LA PAMPA (DGCLP). 2016. http://www.catastro.lapampa.gov.ar/. Libros azules http:// www.catastro. lapampa.gov.ar/index. php/2014-04-23-12-24-49. Libros amarillos (Legajos: exp. 14-35-36, exp. 25-28, exp. 33, exp. 34, exp. 36, exp. 21 , exp. 23, exp. 32 , exp. 24 , exp. 20). 
Último acceso abril 2016.

FARINOS, M. 1919. Mapa del Territorio de La Pampa. Instituto Cartográfico Argentino, Buenos Aires.

IGN. 1886. Atlas. Arturo Seelstrang. Instituto Geográfico Argentino, G Craft, Buenos Aires.

LEFRANCOIS, A. y P. PORRI. 1930. Mapa del Territorio Nacional de La Pampa.

SOLDÁN, M. 1888. Atlas Geográfico de la República Argentina. Félix Lajouane Editor, Buenos Aires. 
